\title{
Direct and Indirect Co-culture of Chondrocytes and Mesenchymal Stem Cells for the Generation of Polymer/ Extracellular Matrix Hybrid Constructs
}

\author{
Erica J. Levorson ${ }^{\mathrm{a}}$, Marco Santoro ${ }^{\mathrm{b}}$, F. Kurtis Kaspera, and Antonios G. Mikos ${ }^{\mathrm{a}, \mathrm{b},{ }^{*}}$ \\ aRice University Department of Bioengineering MS-142, 6100 Main Street Houston, Texas, USA \\ 77005-1892 \\ ${ }^{b}$ Rice University Department of Chemical and Biomolecular Engineering MS-362, 6100 Main \\ Street Houston, Texas, USA 77005-1827
}

\section{Abstract}

\begin{abstract}
In this work, the influence of direct cell-cell contact in co-cultures of mesenchymal stem cells (MSCs) and chondrocytes for the improved deposition of cartilage-like extracellular matrix (ECM) within nonwoven fibrous poly $(\in$-caprolactone) (PCL) scaffolds was examined. To this end, chondrocytes and MSCs were either co-cultured in direct contact by mixing on a single PCL scaffold or via indirect co-culture whereby the two cell types were seeded on separate scaffolds which were then cultured together in the same system either statically or under media perfusion in a bioreactor. In static cultures, the chondrocyte scaffold of an indirectly co-cultured group generated significantly greater amounts of glycosaminoglycan and collagen than the direct coculture group initially seeded with the same number of chondrocytes. Furthermore, improved ECM production was linked to greater cellular proliferation and distribution throughout the scaffold in static culture. In perfusion cultures, flow had a significant effect on the proliferation of the chondrocytes. The ECM contents within the chondrocyte containing scaffolds of the indirect co-culture groups either approximated or surpassed the amounts generated within the direct coculture group. Additionally, within bioreactor culture there were indications that chondrocytes had an influence on the chondrogenesis of MSCs as evidenced by increases in cartilaginous ECM synthetic capacity. This work demonstrates that it is possible to generate PCL/ECM hybrid scaffolds for cartilage regeneration by utilizing the factors secreted by two different cell types, chondrocytes and MSCs, even in the absence of juxtacrine signaling.
\end{abstract}

\section{Keywords}

Cartilage tissue engineering; co-culture; extracellular matrix; chondrocyte; mesenchymal stem cell

\footnotetext{
(C) 2013 Acta Materialia Inc. Published by Elsevier Ltd. All rights reserved.

*Corresponding author: Antonios G. Mikos, Ph.D. Rice University Department of Bioengineering - MS142 P.O. Box 1892 Houston, Texas, USA 77005 mikos@ rice.edu Tel: +1 713 348-4204 Fax: +1 713 348-4244.

Publisher's Disclaimer: This is a PDF file of an unedited manuscript that has been accepted for publication. As a service to our customers we are providing this early version of the manuscript. The manuscript will undergo copyediting, typesetting, and review of the resulting proof before it is published in its final citable form. Please note that during the production process errors may be discovered which could affect the content, and all legal disclaimers that apply to the journal pertain.

Disclosures

No competing financial interests exist.
} 


\section{Introduction}

The tissue engineering paradigm focuses on the combination of cells, scaffolds, and signals with the ultimate goal of regenerating functional tissues. Currently, a large proportion of efforts are focused on the development of bioactive materials that serve as a scaffold, providing structural and mechanical support, and also deliver the bioactive signals to direct cellular differentiation and tissue growth [1-5]. One approach to developing such bioactive materials is to utilize components of the extracellular matrix (ECM) that comprises a particular tissue as a signaling component in an implantable scaffold for the purpose of directing the regeneration of the tissue of interest.

Cartilage, a tissue which is comprised of relatively few cells, is highly dependent on the ECM for form and function. Furthermore, repair of this tissue in the absence of bioactive signals oftentimes results in the repair of a defect with fibrocartilage which is inferior in compressive strength compared to articular cartilage [6, 7]. As such previous efforts have focused on creating scaffolds composed of processed excised cartilage, scaffolds composed solely of isolated collagen and/or glycosaminoglycans (GAGs), as well as engineered threedimensional scaffolds coated with cartilaginous ECM components [5, 8-13]. ECM coated polymeric scaffolds garner the benefits of both the natural and synthetic components. The polymeric scaffold provides the strength and durability to support tissue development while the ECM coating acts as a bioactive signal providing necessary cues to direct regeneration by the host's cells upon implantation. Previous work has shown that chondrocytes cultured on a scaffold under flow perfusion are capable of depositing cartilage-like ECM within the scaffold that is effective at inducing the chondrogenic differentiation of MSCs [14].

As potential for the isolation of chondrocytes from healthy cartilage is limited and because chondrocytes often dedifferentiate upon expansion, other culture methods have been explored to effectively generate cartilage-like ECM in vitro while reducing the number of chondrocytes needed. One such method is by utilizing co-cultures of chondrocytes and MSCs to generate similar quantities of cartilage-like ECM as cultures of chondrocytes alone $[15,16]$. Various studies have shown that co-culturing MSCs with chondrocytes leads to increased chondrogenic gene expression and ECM deposition when cultured both in direct cell-cell contact or separated by a barrier, such as a Transwell@ membrane, or in conditioned media systems [17-21]. These phenotypic changes are considered to be the result of signaling via direct cell to cell contacts [17, 18,21], as well as secreted factors generated by MSCs and chondrocytes [19, 22]. Many studies confirm the secretion of cytokines and growth factors from MSCs exhibiting anti-inflammatory effects in addition to an increase in matrix production by chondrocytes [23-25] while some studies have elucidated positive chondroinduction of MSCs co-cultured with chondrocytes shown to produce growth factors, MMPs, and parathyroid hormone related protein [20, 26, 27]. Previous work showed that a 1:1 ratio of chondrocytes to MSCs was capable of producing similar quantities of cartilage-like ECM as cultures of chondrocytes alone and that the ECM exhibited a similar chondroinductive effect on MSCs as polymer/ECM scaffolds generated using cultures of chondrocytes [15, 28].

The objective of this study was to examine the necessity of direct cell-cell contact in cocultures of MSCs and chondrocytes for the improved deposition of a cartilage-like ECM coating, as defined by an increase in GAG and collagen deposition, within nonwoven fibrous poly $(\in$-caprolactone) (PCL) scaffolds. The hypothesis was that matrix production by chondrocytes co-cultured in direct contact with MSCs would differ in the deposition of cartilage-like ECM from indirectly co-cultured groups due to a potential combined effect of juxtacrine and paracrine signaling. This hypothesis was tested by culturing chondrocytes and MSCs in direct contact by mixing on the same PCL scaffold as well as in indirect co- 
cultures by seeding the two cell types on two separate scaffolds which were then cultured together in the same system. Utilizing both static and perfused culture conditions separately, PCL/ECM construct generation was then characterized by quantifying GAG and collagen contents as well as through imaging the distribution of cells and matrix throughout the scaffold via histology and scanning electron microscopy.

\section{Materials and methods}

\subsection{Scaffold formation}

Non-woven fibrous poly $(\epsilon$-caprolactone) (PCL) scaffolds were fabricated by electrospinning using previously described methods [15]. 18\% (w/w) PCL was first dissolved in a 5:1 (v/v) solution of chloroform: methanol and expelled at a flowrate of 25 $\mathrm{mL} / \mathrm{hr}$ into an electric field formed by a voltage source with $30 \mathrm{kV}$ applied voltage. The collector plate was placed at a distance of $36 \mathrm{~cm}$ from the $16 \mathrm{G}$ needle. Following fabrication, scanning electron microscopy (SEM) (FEI Quanta 400 ESEM FEG, FEICo, Hillsboro, OR) was employed to examine fiber morphology as well as to measure the average fiber size for each mat generated. This was achieved by taking a total of 45 measurements from 3 different locations on the mat using the manufacturer supplied software from which the average and standard deviation were calculated. Electrospun mats 1 $\mathrm{mm}$ thick with an average fiber diameter of $8.5 \mu \mathrm{m}$ and a standard deviation of $1.2 \mu \mathrm{m}$ were die cut into $3 \mathrm{~mm}$ diameter disks and used for the following cellular studies. The average porosity of the scaffolds was $91 \%$ as determined by mercury porosimetry using previously described methods [29].

\subsection{Cell isolation and expansion}

For this study, a previously validated xenogeneic co-culture model using rabbit MSCs and bovine chondrocytes was utilized $[16,30]$. Bovine chondrocytes were isolated from the femoral condyles of 7-10 day old male calves obtained from Research 87 (Research 87, Boston, MA). Following a previously established protocol [14, 15, 31], the chondrocytes from four legs were isolated via a $16 \mathrm{hr}$ incubation with $0.2 \mathrm{wt} \%$ collagenase type II (Worthington, Lakewood, NJ) in culture medium then pooled and cryopreserved. Before seeding scaffolds for both static and perfusion studies, the chondrocytes were first expanded for 7 days in chondrocyte growth medium consisting of DMEM (Invitrogen, Carlsbad, CA), $10 \%$ fetal bovine serum (FBS), $10 \mathrm{mM}$ HEPES buffer (Gibco, Grand Island, NY), 1\% nonessential amino acids (Gibco, Grand Island, NY), $0.28 \mathrm{mM}$ ascorbic acid (Sigma, St Louis, MO), $0.4 \mathrm{mM}$ L-proline (Sigma, St Louis, MO), and 1\% penicillin-streptomycin-fungizone (Gibco, Grand Island, NY).

All rabbit MSC isolations were approved by the Rice University Institutional Animal Care and Use Committee and were in compliance NIH Guide for Care and Use of Laboratory Animals. Rabbit MSCs were isolated from the bone marrow of male New Zealand White rabbits weighing 0.9-1.2 $\mathrm{kg}$ using an established procedure [14, 15, 28, 32]. After approximately 2 weeks of expansion following isolation, the MSCs from 6 rabbits were pooled and cryopreserved. Prior to scaffold seeding the MSCs were removed from cryopreservation and expanded to passage 3 in general growth medium consisting of DMEM (Invitrogen, Carlsbad, CA), 10\% FBS, and 1\% penicillin-streptomycin-fungizone (Gibco, Grand Island, NY).

\subsection{Cell seeding and culture}

PCL scaffolds $(\varnothing=3 \mathrm{~mm})$ were pressfit into polycarbonate seeding cassettes and ethylene oxide sterilized for $12 \mathrm{hr}$. Following sterilization the scaffolds were pre-wet with a decreasing ethanol gradient (100\% - 35\%) and then rinsed with sterile PBS. After pre- 
wetting the scaffolds were incubated overnight in general growth medium to facilitate cellular attachment.

The expanded MSCs and chondrocytes were then trypsinized and five cell seeding solutions were prepared. The same cell solutions were used to seed scaffolds for groups AC 35 and iAC 35 as well as MSC 35 and iMSC 35 (Table 1). In this work, iAC and iMSC indicate indirect co-cultured scaffolds containing chondrocytes and MSCs respectively. Additionally, dAC:MSC indicates the direct co-culture group with chondrocytes and MSCs seeded together on the same scaffold (Figure 1a and Table 1). The number following the group description indicates the initial seeding density. For the iAC 35 and iMSC 35 groups, 35,000 total cells/scaffold were seeded to control for the total cell number seeded on dAC:MSC scaffolds. Similarly, the seeding density of the iAC 17.5 and iMSC 17.5 groups was intended to control for the number of chondrocytes or MSCs seeded initially on the dAC:MSC scaffolds.

For static studies, chondrocyte growth medium was added to the culture wells after a $2 \mathrm{hr}$ pre-attachment period. After $24 \mathrm{hrs}$, the seeded scaffolds were removed from the cassettes and placed in ultra low attachment 24 well plates. Prior to removing the scaffolds from the cassettes, the culture wells were divided in half with a vertical piece of polypropylene mesh (Spectrum Labs, Rancho Dominguez, CA) to spatially separate the iAC and iMSC scaffolds of similar seeding densities while allowing for the transfer of media and soluble factors throughout the entire culture well. The scaffolds were then cultured in chondrocyte growth medium for 14 and 21 days on a shaker table to facilitate media mixing with half of the medium volume changed every 3 days. The experimental groups and culture arrangements are described further in Figure 1 and Table 1.

Dynamic cultures were performed in a flow perfusion bioreactor according to previous studies in our laboratory [31,33]. Briefly, a cassette containing 10 scaffolds of $3 \mathrm{~mm}$ in diameter was placed in each flow perfusion bioreactor unit, together with $50 \mathrm{~mL}$ of chondrocyte growth medium. The chondrocyte containing scaffolds (AC 35, dAC:MSC, iAC 35, and iAC 17.5) as well as MSC 35 were subject to direct flow perfusion with each group in a separate bioreactor. Following the $24 \mathrm{hr}$ attachment period, iMSC 35 and iMSC 17.5 scaffolds, 10 each, were added as free scaffolds to the media reservoir above the corresponding perfused iAC scaffolds within the perfusion cassettes (Figure 1b). For the bioreactors containing the groups AC 35, MSC 35, and dAC:MSC, 10 blank PCL scaffolds were included above the perfusion cassette to control for flow aberrations that may be the result of the free scaffolds. A stainless steel mesh disk was placed atop the perfusion cassette to prevent the free floating scaffolds from interfering with media flow (Figure 1b). One third of the media was changed every 3 days. The scaffolds were cultured for 5, 10, and 14 days under continuous perfusion at a flow rate of $0.01 \pm 0.001 \mathrm{~mL} / \mathrm{min}(1.4 \mathrm{~mm} / \mathrm{min}$ considering system geometry) through each scaffold.

\subsection{Biochemical assays}

For both static and perfused studies, at the end of each culture period scaffolds were removed from the culture medium and rinsed with PBS 2 times. Two scaffolds were then pooled in $500 \mu \mathrm{L}$ of proteinase $\mathrm{K}$ and incubated for $16 \mathrm{hrs}$ at $56^{\circ} \mathrm{C}$ to digest the samples (static, $\mathrm{n}=4$; perfusion, $\mathrm{n}=8$ ) as described previously [14, 15, 29]. To facilitate the removal of DNA and ECM components from the scaffold, three freeze-thaw-sonicate cycles were performed.

Scaffold cellularity was determined by quantifying scaffold DNA content using a PicoGreen DNA assay kit (Molecular Probes, Eugene, OR) described earlier [15, 28, 29]. Using the PicoGreen kit, the concentration of double stranded DNA within the proteinase K digest was 
quantified by excitation at $490 \mathrm{~nm}$ and emission detected at $520 \mathrm{~nm}$. All samples and standards were run in duplicate.

ECM production was quantified utilizing the 1,9-dimethymethylene blue and hydroxyproline colorimetric assays measuring GAG and total collagen contents respectively according to established protocols [14, 29, 34, 35]. For GAG quantification, $25 \mu \mathrm{L}$ of digested sample or chondroitin sulfate standard was treated with $205 \mu \mathrm{L} \mathrm{1,9-}$ dimethymethylene blue (Sigma, St Louis, MO ) and absorbance was measured at $520 \mathrm{~nm}$ with samples run in duplicate. For the hydroxyproline assay, $100 \mu \mathrm{L}$ of proteinase $\mathrm{K}$ digested sample was hydrolyzed at $115^{\circ} \mathrm{C}$ with $100 \mu \mathrm{L} 12 \mathrm{M} \mathrm{HCl}$ for $4 \mathrm{hr}$ and then evaporated under nitrogen flow. The hydrolyzed samples were then rehydrated with water and analyzed in duplicate by incubation with $50 \mu \mathrm{L}$ chloramine-T (Sigma, St Louis, MO) and $50 \mu \mathrm{L} p$-dimethylaminobenzaldehyde (Sigma, St Louis, MO) at $60^{\circ} \mathrm{C}$ for $30 \mathrm{~min}$ [36]. The absorbance of the samples and hydroxyproline standards were measured at $570 \mathrm{~nm}$.

\subsection{Histology}

After each static and perfused culture period, samples $(n=2)$ were rinsed twice with PBS and fixed in $10 \%$ buffered formalin at $4^{\circ} \mathrm{C}$ overnight. The fixed samples were then dehydrated using an increasing ethanol gradient, stored in $100 \%$ ethanol at $4^{\circ} \mathrm{C}$ overnight, then placed in HistoPrep freezing media (Fisher, Fair Lawn, NJ) for at least $24 \mathrm{hr}$. Finally the samples were embedded in freezing media and $8 \mu \mathrm{m}$ sections were cryosectioned (CM1850, Leica Microsystems, Bannockburn, IL) and affixed to glass Superfrost Plus microscope slides (VWR, Batavia, IL). The sections were then incubated on a slide warmer for 7 days at $45^{\circ} \mathrm{C}$.

Picro sirius red staining was utilized to examine the distribution of ECM components within the scaffold, specifically collagen. Using previously established staining protocols, the sections were hydrated and then treated with $1 \mathrm{~g} / \mathrm{L}$ Direct Red 80 (Sigma Aldrich, St Louis, MO) in saturated aqueous picric acid [15, 28, 34]. After an hour, the sections were clarified twice with $0.5 \%$ acetic acid. Cellular localization was visualized by hydrating the section then staining with $1 \mathrm{~g} / \mathrm{L}$ Nuclear Fast Red (Sigma Aldrich, St Louis, MO) in 50 g/L aluminum sulfate for $5 \mathrm{~min}$ then rinsing with water. Following staining, sections were imaged via light microscopy (Zeiss AxioImager.Z2, Göttingen, Germany) coupled with a digital camera (Zeiss AxioCamMRc5, Göttingen, Germany) correcting exposure and white balance using the manufacturer supplied software.

\subsection{Scanning electron microscopy}

The surface morphology of the scaffolds was imaged using scanning electron microscopy to examine the progression of ECM and cells on the surface of the scaffold. Scaffolds from each time point $(\mathrm{n}=2)$ were fixed for $1 \mathrm{hr}$ in $2.5 \%$ glutaraldehyde and dried using an increasing ethanol gradient. Samples were then sputtercoated with $20 \mathrm{~nm}$ of gold (Denton Desk V, Moorestown, NJ) and SEM (FEI Quanta 400 ESEM FEG, FEICo, Hillsboro, OR) images were obtained.

\subsection{Statistical analysis}

The results of the biochemical assays are presented as means \pm standard deviation. A twoway ANOVA was utilized to determine significant differences in DNA and ECM contents of the scaffolds as the result of culture duration and cell population. Tukey's HSD testing was used to perform multiple comparisons when significance via ANOVA was determined. For all statistical analyses, significance was defined as $\mathrm{p}<0.05$. 


\section{Results}

\subsection{Surface morphology}

SEM was utilized to examine the surface morphology of the scaffolds with the progressing deposition of ECM and proliferation of the cells within the different groups (Figure 2 and Supplementary Figure 1). For static samples, within 14 days the surfaces of scaffolds cultured with chondrocytes were mostly covered with ECM and cells (Supplementary Figure $1(a)-(d))$. It took between 14 and 21 days for the deposits of ECM and cells to primarily obscure the underlying PCL fibers within these static chondrocyte containing groups. For the static MSC-only scaffolds, only the iMSC 17.5 group displayed prominent evidence of ECM and cells on the scaffold surface in 14 days (Supplementary Figure $1(e)-(g)$ ). However, within 21 days the surfaces of all three static MSC-only scaffolds were mostly covered with cells and ECM. On the scaffolds cultured in the bioreactor, the surface of the chondrocyte containing groups were completely covered with cells and ECM in 5 days except for the iAC 35 group which was covered within 10 days (Figure $2(a)-(d)$ ). For the MSC-only scaffolds, all three MSC-only scaffolds were covered with cells and ECM by day 14 (Figure $2(e)-(g))$. Furthermore, as it becomes more difficult to discern the underlying PCL fibers the surface morphology of the cell and ECM coating becomes more rough in appearance for both static and bioreactor cultures (Supplementary Figure $1 \mathrm{~d} 21(a)$ and $(d)$; Figure $2 \mathrm{~d} 10(c)$ and $\mathrm{d} 14(a))$.

\subsection{Histological characterization}

Within scaffolds cultured statically, Nuclear Fast Red staining for cell nuclei shows that a great proportion of the cells were located at the surface of the scaffolds at both time points (Supplementary Figure 2). However, among all of the static scaffolds the 3 groups seeded with chondrocytes alone displayed the greatest distribution of cells and matrix within the scaffold at both time points as indicated by Nuclear Fast Red and Picro Sirius Red staining respectively (Figure 3 and Supplementary Figure $2(a)-(c)$ ). Comparing between the $\mathrm{dAC}: \mathrm{MSC}$ and iAC groups, there was little difference in cell localization or matrix distribution at 14 days of static culture. However, by 21 days of culture the two indirectly co-cultured iAC groups display more intense staining for cells and matrix within the scaffold than the direct co-culture scaffold, dAC:MSC (Figure 3 and Supplementary Figure $2(b)$ $(d)$ ). Among the AAC:MSC and iMSC groups in static culture, there was not a marked difference in cell or matrix distribution at 21 days of culture even though there was a somewhat more intense staining for collagen within the dAC:MSC scaffolds at 14 days. At 21 days of static culture, the iMSC 35 group displays greater amounts of collagenous matrix within the scaffold than the MSC 35 or iMSC 17.5 groups (Figure $3(e)-(g)$ ). This difference among the MSC groups, was not observed when examining cell localization (Supplementary Figure $2(e)-(g))$.

Within the bioreactor cultures, staining for cells and ECM became more pronounced as the culture duration increased from 5 to 14 days. Among the day 14 bioreactor scaffolds, the AC 35 and iAC 35 groups exhibited the greatest amount of staining for collagen both at the surface of the scaffolds as well as in the interior (Figure $4(a)$ and $(b)$ ). For all 3 time points, the chondrocyte containing groups had greater amounts of collagen deposition than the MSC-only samples. Among direct and indirectly co-cultured scaffolds, there was an increased staining for collagen within the iAC 35 scaffolds compared to the directly cocultured dAC:MSC scaffolds at day 14 (Figure $4(b)$ and $(d)$ ). Although, the direct coculture scaffolds showed greater collagen deposition at the first two time points compared to the indirectly co-cultured MSC scaffolds, iMSC 35 and iMSC 17.5 (Figure $4(d),(f)$ and $(g)$ ). In general, the staining for cell localization showed very minor differences amongst the bioreactor groups especially at the latest time point. All of the scaffolds displayed a dense 
coating of cells at the surface of the scaffolds at day 14. There were more cells located inside the 3 groups seeded with chondrocytes alone than inside the 3 MSC-only groups (Supplementary Figure 3 d14). The differences in cell localization were more pronounced at the earlier time points where the 4 chondrocyte containing groups exhibited more intense Nuclear Fast Red staining than the 3 MSC-only groups.

\subsection{Biochemical characterization-Static}

Cellularity, as determined by DNA content, increased gradually throughout the culture period for the four static chondrocyte containing groups (Figure 5a "x" and "*”). In contrast, when cultured statically little proliferation was observed within the three MSC-only groups. Within the d21 static samples, iAC 17.5 displayed greater DNA contents than the dAC:MSC group despite initially being seeded with fewer cells (Figure 5a "\#”).

As with cell proliferation, the deposition of GAGs within the four chondrocyte containing groups gradually increased with greater culture duration (Figure $6 a$ "x" and “*”). The iAC 17.5 scaffolds were the only group to display a significant increase in GAG content between d14 and d21 in addition to producing significantly greater GAG contents than the direct coculture group, dAC:MSC. Furthermore, the indirect co-culture AC scaffolds generated significantly greater amounts of GAGs at d21 than the similarly cultured MSC scaffolds (Figure $6 a$ “ $¥$ ”). When GAG content was normalized to the DNA content of the scaffolds, there was no significant difference in GAG synthetic capacity observed among the statically cultured scaffolds except for the d21 MSC 35 group which was found to be significantly greater than the day 0 scaffolds (Figure $6 b$ "x").

Similar trends were observed in collagen production within the statically cultured groups as were detected in scaffold cellularity and GAG content. Specifically, at d21 the indirectly cocultured chondrocyte group iAC 17.5 produced significantly greater amounts of collagen than the direct co-culture group, dAC:MSC (Figure 7a “ $\ddagger$ ”). Furthermore, the chondrocyte containing groups produced more collagen at $\mathrm{d} 21$ than the corresponding MSC containing groups cultured similarly. However, there was little difference in collagen synthetic capacity as determined by collagen content normalized to DNA content among the different experimental groups (Figure $7 b$ ).

\subsection{Biochemical characterization-Bioreactor}

As in static cultures, cellularity increased gradually throughout the culture period for the four chondrocyte containing groups cultured in the bioreactor (Figure $5 b$ " $x$ " and "*”"). After 14 days of bioreactor culture, the two indirect co-culture chondrocyte scaffolds and the AC 35 samples exhibited significantly higher cell contents than the direct co-culture group, dAC:MSC (Figure 5b “\#”). Additionally, there was little proliferation observed within the three MSC-only groups throughout the 14 days of bioreactor culture.

At day 14, the chondrocyte-only group and both indirect co-culture groups produced more GAGs than the direct co-culture group, dAC:MSC (Figure 6c “\#”), while no groups contained more GAGs than the dAC:MSC groups at previous time points under flow conditions. The AC scaffolds initially seeded with 35,000 chondrocytes, AC 35 and iAC 35, generated significantly greater amounts of GAGs at d14 than the similarly cultured MSC scaffolds (Figure 6c “:”). Conversely, there was no significant difference in the GAG content of iAC 17.5 and iMSC 17.5 groups after 14 days of bioreactor culture. There was an increase in the deposition of GAGs as culture duration increased for the four chondrocyte containing groups (Figure $6 \mathrm{c}$ " $\mathrm{x}$ " and "**”) with all of the groups containing significantly greater GAG contents than their day 0 counterparts within 10 days of culture except for the 
iAC 35 samples. Conversely, as in static cultures, there were minimal changes in the deposition of GAGs with time by the 3 MSC-only groups in the bioreactor.

While GAG synthetic capacity remained relatively stable in static culture, there were some changes observed within the bioreactor cultures. An increase in GAG synthetic capacity was observed at day 14 within the groups AC 35, iAC 35, and the two indirectly co-cultured MSC groups, iMSC 35 and iMSC 17.5 (Figure 6d "x"). iMSC 17.5 was the only group at an earlier time point to show a significant increase in GAG synthetic capacity.

Samples produced GAGs at a faster rate within the bioreactor than in static cultures as qualitatively determined by the number of day 14 experimental groups to contain significantly greater GAG contents than at initial time points. Specifically, after 14 days of culture under flow conditions 5 groups had significantly greater GAG quantities than day 0 counterparts while none of the static groups were determined to be significantly greater than day 0 in static cultures (Figures 6a and 6c "x"). In general, there were no significant differences observed as the result of initial seeding density within the AC or MSC scaffolds cultured via indirect co-culture in both static and flow conditions. The only exception occurred in bioreactor GAG synthetic capacity at days 5 and 14 between groups iMSC 35 and iMSC 17.5 where iMSC 17.5 displayed a higher capacity for ECM synthesis (Figure $6 d)$.

Scaffolds under flow conditions generated significant increases in collagen contents by 14 days of culture (Figure 7c " $x$ " and “*”). All of the chondrocyte containing groups showed a significant increase in collagen content over day 0 counterparts except for the direct coculture group, dAC:MSC. Moreover, both groups seeded initially with 35,000 chondrocytes/ scaffold, AC 35 and iAC 35, generated significantly greater amounts of collagen than the dAC:MSC group after 14 days of bioreactor culture. Additionally, the AC 35 group, consisting of chondrocytes cultured on PCL alone without any form of co-culture, produced more collagen within the PCL scaffold than all three chondrocyte containing co-culture groups (Figure 7c "\#” and "+"). As with previous results examining matrix production, there was little difference between the three MSC cultured groups in the bioreactor

There was a rather marked increase in collagen synthetic capacity within the bioreactor groups between days 10 and 14 (Figure 7d " $x$ " and "*"). This is somewhat different from the gradual or nonexistent changes in ECM synthetic activity that were observed in static cultures as well as bioreactor GAG synthetic capacity.

\section{Discussion}

This study demonstrated the influence of direct and indirect co-culture of chondrocytes and MSCs on the development of cartilage-like ECM, as defined by an increase in GAG and collagen deposition, within a fibrous PCL scaffold under static and perfused culture conditions. Specifically, the results suggest that polymer/ECM hybrid scaffolds containing GAGs and collagen may be generated by utilizing the factors secreted by two different cell types, chondrocytes and MSCs, even in the absence of direct cell-cell contact. This was demonstrated in both static and perfused cultures. Utilizing cell culture to generate ECM coatings on polymeric scaffolds is a relatively novel approach to producing natural/synthetic hybrid scaffolds. Other methods of fabricating similar hybrid scaffold materials by coating 3D polymer scaffolds with isolated ECM components, such as collagen or GAGs, benefit from well characterized and controlled formulations [8, 12, 13, 37, 38]. However, using cell culture to generate polymer/ECM hybrid scaffolds has the potential to deposit ECM components in physiologically active conformations as well as potentially incorporate growth factors and other morphogens useful for directing tissue regeneration [14, 34, 39]. 
By studying in vitro cell generated polymer/ECM hybrid scaffolds using a variety of cell sources, we have shown previously that flow perfusion, oxygen tension, as well as culture supplements can be useful for the generation of scaffolds for both bone and cartilage regeneration [30, 31, 34, 40-42]. Furthermore, it has been demonstrated that upon devitalization these various polymer/ECM hybrid scaffolds are capable of inducing the osteogenic and chondrogenic differentiation of MSCs [14, 28, 34, 35, 40, 42]. Recent studies have focused on utilizing co-cultures of chondrocytes and MSCs to generate cartilage-like ECM within a scaffold capable of directing cartilage regeneration. In these studies, results indicated that in direct co-cultures of chondrocytes and MSCs there is a stimulatory effect on the production of cartilage-like ECM $[15,16]$. Specifically, co-cultures of chondrocytes and MSCs in equal proportions generated constructs with similar quantities and spatial distributions of cartilage-like ECM as constructs produced by chondrocytes alone [15]. Furthermore, as compared to untreated PCL controls, devitalized PCL/ECM scaffolds generated by culturing with a 1:1 chondrocyte to MSC ratio had a positive effect on the aggrecan and collagen type II gene expression of MSCs [28]. This echoes previous results which indicated that PCL/ECM generated by chondrocytes in a flow perfusion bioreactor led to a decrease in collagen type I expression of reseeded MSCs and an increase in the capacity for the MSCs to produce cartilage-like ECM [14]. The work described here functioned to further investigate the fabrication of these co-culture generated chondroinductive PCL/ECM constructs by determining the benefit of direct versus indirect co-culture in the capacity to form significant amounts of GAGs and collagen distributed throughout the scaffold. Specifically, we hypothesized that direct cell-cell contact as well as cell-secreted trophic factors may influence ECM production within the fibrous scaffolds.

In static cultures, no difference in cellularity or ECM content was determined between the chondrocyte-only group seeded with 35,000 chondrocytes, AC 35, and the indirect coculture group, iAC 17.5, that was seeded with half as many chondrocytes initially. Conversely, the indirectly co-cultured group iAC 17.5 led to significant increases in cellularity and ECM content over the direct co-culture samples, dAC:MSC, in 21 days of static culture even though it was seeded with the same number of chondrocytes initially (Figures 5a, 6a, and 7a “\#”). Upon examining ECM synthetic capacity within the chondrocyte containing groups, there was very little difference among the various experimental groups. As such, it follows that in static cultures the ultimate production of ECM within the PCL scaffolds was highly dependent on the total number of cells present to secrete matrix throughout the culture duration. Furthermore, it appears as though the cellularity at the later time point was not dependent on the initial seeding density as the groups seeded with half the amount of cells initially, iAC 17.5 and iMSC 17.5, ultimately attained similar DNA contents as the other groups seeded with similar cell types but at twice the initial seeding density. One potential explanation for this behavior is that within these fibrous scaffolds cells may proliferate to the point of overcrowding within 21 days of static culture possibly inducing a contact inhibition-like response [43, 44].

SEM of scaffolds cultured statically for 14 and 21 days shows the surface is covered and appears similar for chondrocyte seeded scaffolds cultured statically (Supplementary Figure 1). Based on histology, there is a large amount of cells and ECM at the surface of the scaffolds as told by SEM. However differences among the groups lie within the interior of the scaffolds where the scaffolds seeded solely with chondrocytes, AC 35, iAC 35, and iAC17.5, show thorough distributions of cells and matrix within the scaffolds when dAC:MSC and MSC scaffolds are somewhat sparse in comparison (Figure 3 and Supplementary Figure 2). When compared to the DNA and ECM contents of the scaffolds, it follows that more thorough distributions of cells and ECM throughout the structure would be correlated to higher quantities of DNA and ECM within the scaffold. This interplay between cellular distribution within a scaffold and its influence on proliferation and tissue 
development is oftentimes investigated under flow perfusion which has shown to lead to improved cellular distributions over static culture [44-46].

In an earlier co-culture study conducted in serum-free media with TGF- $\beta 3$ supplementation, it was found that while MSCs gradually decrease in proportion throughout the culture period the co-cultures composed of as much as 75\% MSCs initially led to increases in GAG content and improved collagen type II expression over chondrocyte cultures [16]. Additionally, based on rtPCR using species specific primers it was determined that within these direct cocultures the majority of the chondrogenic expression originated from the chondrocytes; suggesting that the MSCs had a stimulatory effect on the chondrocytes [16]. For purpose of our investigation, flow perfusion bioreactor culture was utilized to not only improve general gas and nutrient transport properties [33, 46, 47], but also to flush any factors secreted by the MSCs through the chondrocyte containing scaffolds in order to further examine the potential response due to paracrine signaling within indirect co-cultures. Moving from static to perfused culture, shorter culture durations were necessary in the bioreactor as extended periods of perfusion culture oftentimes fail due to cell proliferation and matrix deposition blocking the media flow path. Alternately, in order to observe significant differences in static culture longer culture durations were needed. In addition, as different media volumes were necessary for the two different culture conditions, statistical analyses comparing the proliferation and matrix production in static versus bioreactor culture were not performed.

As in static culture, the cartilage-like ECM synthetic capacities of the three chondrocyte containing co-culture groups showed no statistically significant differences under flow conditions (Figures $6 d$ and $7 d$ ). However, unlike in static culture the two indirect co-culture groups, iAC 35 and iAC 17.5, resulted in significant increases in cellularity by day 14 over the direct co-culture group with iAC 35 also showing a significant increase in ECM content over the direct co-culture group. As the direct co-cultures did not surpass the matrix production by the indirect co-cultures, it may possible to generate PCL/ECM hybrid scaffolds for cartilage regeneration by utilizing the factors secreted by two different cell types, chondrocytes and MSCs, without juxtacrine signaling. Although decellularized PCL/ ECM hybrid scaffolds generated similarly have shown chondrogenic effects on reseeded MSCs in the form of increased aggrecan and collagen type II expression [28], to further understand the interplay between these two cell types and the influence on ECM production the specific composition of the ECM must be investigated further in future studies.

A previous study performed by Bian et al, showed that mixed populations of MSCs and chondrocytes encapsulated in hydrogels resulted in improved matrix production compared to MSC and chondrocyte-only controls. However, there was no benefit of indirect co-cultures of MSC-only encapsulated hydrogels cultured together with chondrocyte-only encapsulated hydrogels [21]. It may be possible that hydrogel encapsulation could constrain the diffusion of secreted factors and cellular infiltration [48]. As such, this work investigated the influence of direct and indirect co-culture on matrix production in a fibrous mesh scaffold as well as with perfused flow in an effort to improve the dispersion of secreted signals. This study also utilized a previously validated xenogeneic co-culture model $[15,16,30]$. In this model, rabbit MSCs were employed in keeping with the regularly used rabbit in vivo model for cartilage engineering [49-52] and bovine chondrocytes were utilized to reduce the number of animals needed due to the limited tissue available for chondrocyte isolation from rabbit condyles. The development of a xenogeneic co-culture model has shown to be not only useful in elucidating some of the positive effects of allogeneic co-culture, but also enables the potential to perform species specific characterization methodologies [16, 22, 24].

Within 14 days of culture under perfused flow all of the experimental groups exhibited approximately similar DNA contents as those observed in equal or greater durations of static 
culture. Furthermore, bioreactor culture led to faster proliferation rates within the chondrocyte containing groups than static culture with significant changes observed at 5 and 10 days as opposed to 21 days in static (Figure 5 "x"). Similar results were observed previously, in that perfusion led to improved proliferation and GAG deposition by chondrocytes over static cultures [31]. While fluid flow had a significant influence on cellular proliferation in the chondrocyte containing groups, there was no significant change in the DNA content of the three MSC-only scaffolds regardless of co-culture. These results suggest that perfusion has more of an influence on chondrocyte proliferation than MSC growth regardless of co-culture. Additionally, the presence of MSCs in indirect co-culture did not appear to have a negative effect on chondrocyte growth which was a potential concern as nutrient limitations have shown to impose growth inhibition [43].

While little proliferation or increase in ECM content was observed within the three MSConly scaffolds in both static and perfused culture, for both iMSC groups the GAG synthetic capacity was higher than their iAC counterparts in the bioreactor (Figure 6d " $¥$ "). There was also a temporal increase in ECM synthetic capacity observed within the MSC indirect coculture scaffolds, iMSC 35 and iMSC 17.5, while no change was observed within the MSConly scaffolds, MSC 35 (Figures 6d and 7d). It is important to note that within the bioreactor design employed here, the indirect MSC scaffolds were not directly perfused with media rather the scaffolds were cultured loosely within the media that would then flow through the chondrocyte containing scaffolds. As no change in ECM anabolic capacity was observed in static cultures or the media perfused MSC-only scaffolds, it follows that cellular secreted factors from indirect co-culture resulted in improvement in the ECM production by MSCs. Additionally, qualitatively indirect co-culture paired with bioreactor culture resulted in improved cellular localization and ECM distribution throughout the MSC-only scaffolds over static culture (Figures 3-4 and Supplementary Figures 2-3 d14 $(e)-(g)$ ). As previous reports have focused on the effect of MSCs on matrix production by chondrocytes this alteration in MSC ECM synthetic capacity within indirect co-cultures was unexpected [16, $24,53]$. Future work is needed to more thoroughly investigate the influence of direct and indirect co-culture on the differentiation state of MSCs. It is possible that direct co-culture has masked some of the subtle positive effects co-culture with chondrocytes has on MSCs. Various studies have indicated that MSCs secrete trophic factors including IGF-1, TGF- $\beta$, and BMP-2 which are known to have a positive effect on the chondrocytic phenotype [25, 54-56]. Conversely, this and other studies have shown that chondrocytes also influence the chondrogenic differentiation of MSCs, as evidenced by improved ECM synthetic capacity, through the secretion of paracrine factors such as parathyroid hormone-related protein [20, $25,27,57,58]$. In some cases, when cultured in separated co-culture with chondrocytes via Transwell@ plates or treated with chondrocyte conditioned media MSCs have shown improved chondrogenesis and reduced hypertrophy $[17,19,20,57]$. From these various results, it appears that the relationship within chondrocyte and MSC co-cultures is complex and continued mechanistic work needs to be carried out in an effort to fully understand the communication occurring between these two cell types in an effort to improve tissue regeneration.

\section{Conclusions}

The objective of this study was to examine the influence of direct cell-cell contact in cocultures of MSCs and chondrocytes on the deposition of cartilage-like ECM within nonwoven fibrous PCL scaffolds. In static culture, the indirectly co-cultured chondrocytes with the lower seeding density led to significant increases in cellularity and ECM content over the direct co-cultures of chondrocytes and MSCs despite being seeded with the same number of chondrocytes initially. Within static cultures, the ultimate production of ECM within the PCL scaffolds was highly dependent on the number of cells present to secrete 
matrix and their distribution in the scaffold. However, ECM production was not contingent on the initial number of cells seeded. Perfused culture has previously shown to result in improved distributions in 3D scaffolds, and was utilized here to examine the interplay between direct and indirect co-culture in another manner. Fluid flow had more of a significant effect on the proliferation of chondrocytes than on MSCs. Additionally, within bioreactor culture there were indications that chondrocytes had an influence on the chondrogenesis of MSCs as evidenced by increases in cartilaginous ECM synthetic capacity. These results indicate that polymer/ECM hybrid scaffolds may be formed by utilizing the factors secreted by two different cell types, chondrocytes and MSCs, even in the absence of direct cell-cell contact.

\section{Supplementary Material}

Refer to Web version on PubMed Central for supplementary material.

\section{Acknowledgments}

This work was funded by the National Institute of Health (R01AR57083).

\section{References}

1. Jansen JA, Vehof JWM, Ruhé PQ, Kroeze-Deutman H, Kuboki Y, Takita H, et al. Growth factorloaded scaffolds for bone engineering. J Control Release. 2005; 101:127. [PubMed: 15588899]

2. Park H, Temenoff JS, Holland TA, Tabata Y, Mikos AG. Delivery of TGF-[beta]1 and chondrocytes via injectable, biodegradable hydrogels for cartilage tissue engineering applications. Biomaterials. 2005; 26:7095-103. [PubMed: 16023196]

3. Harrison BS, Eberli D, Lee SJ, Atala A, Yoo JJ. Oxygen producing biomaterials for tissue regeneration. Biomaterials. 2007; 28:4628. [PubMed: 17681597]

4. Bouten CVC, Dankers PYW, Driessen-Mol A, Pedron S, Brizard AMA, Baaijens FPT. Substrates for cardiovascular tissue engineering. Adv Drug Deliv Rev. 2011; 63:221-41. [PubMed: 21277921]

5. Solchaga LA, Gao J, Dennis JE, Awadallah A, Lundberg M, Caplan AI, et al. Treatment of Osteochondral Defects with Autologous Bone Marrow in a Hyaluronan-Based Delivery Vehicle. Tissue Eng. 2002; 8:333-47. [PubMed: 12031121]

6. Calandruccio RA, Gilmer WS JR. Proliferation, Regeneration, and Repair of Articular Cartilage of Immature Animals. J Bone Joint Surg Am. 1962; 44:431-55.

7. Weisser J, Rahfoth B, Timmermann A, Aigner T, Bräuer R, von der Mark K. Role of growth factors in rabbit articular cartilage repair by chondrocytes in agarose. Osteoarthr Cartil. 2001; 9:S48-S54. [PubMed: 11680688]

8. Benders KEM, Weeren PRv, Badylak SF, Saris DBF, Dhert WJA, Malda J. Extracellular matrix scaffolds for cartilage and bone regeneration. Trends Biotechnol. 2013; 31:169-76. [PubMed: 23298610]

9. Matthews JA, Boland ED, Wnek GE, Simpson DG, Bowlin GL. Electrospinning of Collagen Type II: A Feasibility Study. J Bioact Compat Polym. 2003; 18:125-34.

10. Bhosale AM, Richardson JB. Articular cartilage: structure, injuries and review of management. $\mathrm{Br}$ Med Bull. 2008; 87:77-95. [PubMed: 18676397]

11. Chen W-C, Yao C-L, Wei Y-H, Chu IM. Evaluating osteochondral defect repair potential of autologous rabbit bone marrow cells on type II collagen scaffold. Cytotechnology. 2011; 63:1323. [PubMed: 20972620]

12. Wollenweber M, Domaschke H, Hanke T, Boxberger S, Schmack G, Gliesche K, et al. Mimicked Bioartificial Matrix Containing Chondroitin Sulphate on a Textile Scaffold of Poly(3hydroxybutyrate) Alters the Differentiation of Adult Human Mesenchymal Stem Cells. Tissue Eng. 2006; 12:345-59. [PubMed: 16548693] 
13. Hsu, S-h; Yen, H-J.; Tseng, C-S.; Cheng, C-S.; Tsai, C-L. Evaluation of the growth of chondrocytes and osteoblasts seeded into precision scaffolds fabricated by fused deposition manufacturing. J Biomed Mater Res B Appl Biomater. 2007; 80B:519-27. [PubMed: 16862556]

14. Liao J, Guo X, Grande-Allen KJ, Kasper FK, Mikos AG. Bioactive polymer/extracellular matrix scaffolds fabricated with a flow perfusion bioreactor for cartilage tissue engineering. Biomaterials. 2010; 31:8911-20. [PubMed: 20797784]

15. Levorson EJ, Mountziaris PM, Hu O, Kasper FK, Mikos AG. Cell Derived Polymer/Extracellular Matrix Composite Scaffolds for Cartilage Regeneration, Part 1: Investigation of Co-cultures and Seeding Densities for Improved Extracellular Matrix Deposition. Tissue Eng Part C Methods. DOI: 10.1089/ten.TEC.2013.0286.

16. Meretoja VV, Dahlin RL, Kasper FK, Mikos AG. Enhanced chondrogenesis in co-cultures with articular chondrocytes and mesenchymal stem cells. Biomaterials. 2012; 33:6362-9. [PubMed: 22695067]

17. Chen W-H, Lai M-T, Wu ATH, Wu C-C, Gelovani JG, Lin C-T, et al. In vitro stage-specific chondrogenesis of mesenchymal stem cells committed to chondrocytes. Arthritis Rheum. 2009; 60:450-9. [PubMed: 19180515]

18. Hendriks J, Riesle J, van Blitterswijk CA. Co-culture in cartilage tissue engineering. J Tissue Eng Regen Med. 2007; 1:170-8. [PubMed: 18038408]

19. Hwang NS, Varghese S, Puleo C, Zhang Z, Elisseeff J. Morphogenetic signals from chondrocytes promote chondrogenic and osteogenic differentiation of mesenchymal stem cells. J Cell Physiol. 2007; 212:281-4. [PubMed: 17520697]

20. Fischer J, Dickhut A, Rickert M, Richter W. Human articular chondrocytes secrete parathyroid hormone-related protein and inhibit hypertrophy of mesenchymal stem cells in coculture during chondrogenesis. Arthritis Rheum. 2010; 62:2696-706. [PubMed: 20496422]

21. Bian L, Zhai DY, Mauck RL, Burdick JA. Coculture of Human Mesenchymal Stem Cells and Articular Chondrocytes Reduces Hypertrophy and Enhances Functional Properties of Engineered Cartilage. Tissue Engineering Part A. 2011; 7:1137-45. [PubMed: 21142648]

22. Wu L, Leijten JCH, Georgi N, Post JN, Blitterswijk CAv, Karperien M. Trophic Effects of Mesenchymal Stem Cells Increase Chondrocyte Proliferation and Matrix Formation. Tissue Eng Part A. 2011; 17:1425-36. [PubMed: 21247341]

23. van Buul GM, Villafuertes E, Bos PK, Waarsing JH, Kops N, Narcisi R, et al. Mesenchymal stem cells secrete factors that inhibit inflammatory processes in short-term osteoarthritic synovium and cartilage explant culture. Osteoarthritis and cartilage / OARS, Osteoarthritis Research Society. 2012; 20:1186-96.

24. Wu L, Prins H-J, Helder MN, Blitterswijk CAv, Karperien M. Trophic effects of mesenchymal stem cells in chondrocyte co-cultures are independent of culture conditions and cell sources. Tissue Eng Part A. 2012; 18:1542-51. [PubMed: 22429306]

25. Sekiya I, Vuoristo JT, Larson BL, Prockop DJ. In vitro cartilage formation by human adult stem cells from bone marrow stroma defines the sequence of cellular and molecular events during chondrogenesis. PNAS. 2002; 99:4397-402. [PubMed: 11917104]

26. Lettry V, Hosoya K, Takagi S, Okumura M. Coculture of equine mesenchymal stem cells and mature equine articular chondrocytes results in improved chondrogenic differentiation of the stem cells. Japanese Journal of Veterinary Research. 2010; 58:5-15. [PubMed: 20645581]

27. Ahmed N, Dreier R, Göpferich A, Grifka J, Grässel S. Soluble signalling factors derived from differentiated cartilage tissue affect chondrogenic differentiation of rat adult marrow stromal cells. Cell Physiol Biochem. 2007; 20:665-78. [PubMed: 17762193]

28. Levorson EJ, Hu O, Mountziaris PM, Kasper FK, Mikos AG. Cell Derived Polymer/Extracellular Matrix Composite Scaffolds for Cartilage Regeneration, Part 2: Construct Devitalization and Determination of Chondroinductive Capacity. Tissue Eng Part C Methods. DOI: 10.1089/ten.tec. 2013.0288.

29. Levorson EJ, Sreerekha PR, Chennazhi KP, Kasper FK, Nair SV, Mikos AG. Fabrication and Characterization of Multiscale Electrospun Scaffolds for Cartilage Regeneration. Biomed Mater. 2013; 8:014103. [PubMed: 23353096] 
30. Meretoja VV, Dahlin RL, Wright S, Kasper FK, Mikos AG. The effect of hypoxia on the chondrogenic differentiation of co-cultured articular chondrocytes and mesenchymal stem cells in scaffolds. Biomaterials. 2013; 34:4266-73. [PubMed: 23489925]

31. Dahlin RL, Meretoja VV, Ni M, Kasper FK, Mikos AG. Hypoxia and flow perfusion modulate proliferation and gene expression of articular chondrocytes on porous scaffolds. AIChE J. 2013; 59:3158-66.

32. Guo X, Liao J, Park H, Saraf A, Raphael RM, Tabata Y, et al. Effects of TGF-beta3 and preculture period of osteogenic cells on the chondrogenic differentiation of rabbit marrow mesenchymal stem cells encapsulated in a bilayered hydrogel composite. Acta Biomater. 2010; 6:2920-31. [PubMed: 20197126]

33. Dahlin RL, Meretoja VV, Ni M, Kasper FK, Mikos AG. Design of a High-Throughput Flow Perfusion Bioreactor System for Tissue Engineering. Tissue Eng Part C Methods. 2012; 18:81720. [PubMed: 22468910]

34. Thibault RA, Mikos AG, Kasper FK. Osteogenic differentiation of mesenchymal stem cells on demineralized and devitalized biodegradable polymer and extracellular matrix hybrid constructs. J Biomed Mater Res A. 2013; 101A:1225-36. [PubMed: 23505119]

35. Datta N, Pham QP, Sharma U, Sikavitsas VI, Jansen JA, Mikos AG. In vitro generated extracellular matrix and fluid shear stress synergistically enhance 3D osteoblastic differentiation. PNAS. 2006; 103:2488-93. [PubMed: 16477044]

36. Stegemann H, Stalder K. Determination of hydroxyproline. Clin Chim Acta. 1967; 18:267-73. [PubMed: 4864804]

37. Barnes CP, Pemble CW, Brand DD, Simpson DG, Bowlin GL. Cross-Linking Electrospun Type II Collagen Tissue Engineering Scaffolds with Carbodiimide in Ethanol. Tissue Eng. 2007; 13:1593605. [PubMed: 17523878]

38. Rentsch B, Hofmann A, Breier A, Rentsch C, Scharnweber D. Embroidered and Surface Modified Polycaprolactone-Co-Lactide Scaffolds as Bone Substitute: In Vitro Characterization. Ann Biomed Eng. 2009; 37:2118-28. [PubMed: 19626441]

39. Gomes ME, Bossano CM, Johnston CM, Reis RL, Mikos AG. In vitro localization of bone growth factors in constructs of biodegradable scaffolds seeded with marrow stromal cells and cultured in a flow perfusion bioreactor. Tissue Eng. 2006; 12:177-88. [PubMed: 16499454]

40. Liao J, Guo X, Nelson D, Kurtis Kasper F, Mikos AG. Modulation of osteogenic properties of biodegradable polymer/extracellular matrix scaffolds generated with a flow perfusion bioreactor. Acta Biomater. 2010; 6:2386-93. [PubMed: 20080214]

41. Thibault RA, Mikos AG, Kasper FK. Protein and Mineral Composition of Osteogenic Extracellular Matrix Constructs Generated with a Flow Perfusion Bioreactor. Biomacromolecules. 2011; 12:4204-12. [PubMed: 22040097]

42. Thibault RA, Scott Baggett L, Mikos AG, Kasper FK. Osteogenic Differentiation of Mesenchymal Stem Cells on Pregenerated Extracellular Matrix Scaffolds in the Absence of Osteogenic Cell Culture Supplements. Tissue Eng Part A. 2010; 16:431-40. [PubMed: 19863274]

43. Martin JMM, Smith M, Al-Rubeai M. Cryopreservation and in Vitro Expansion of Chondroprogenitor Cells Isolated from the Superficial Zone of Articular Cartilage. Biotechnol Prog. 2005; 21:168-77. [PubMed: 15903255]

44. Li Y, Ma T, Kniss DA, Lasky LC, Yang S-T. Effects of Filtration Seeding on Cell Density, Spatial Distribution, and Proliferation in Nonwoven Fibrous Matrices. Biotechnol Prog. 2001; 17:935-44. [PubMed: 11587587]

45. Alvarez-Barreto J, Linehan S, Shambaugh R, Sikavitsas V. Flow perfusion improves seeding of tissue engineering scaffolds with different architectures. Ann Biomed Eng. 2007; 35:429. [PubMed: 17216348]

46. Levorson, EJ.; Kasper, FK.; Mikos, AG. Scaffolds - Flow Perfusion Bioreactor Design.. In: Ducheyne, P.; Healy, KE.; Hutmacher, DW.; Kirkpatrick, CJ., editors. Comprehensive Biomaterials. Elsevier; Oxford: 2011. p. 1-11.

47. Bancroft GN, Sikavitsas VI, Mikos AG. Technical Note: Design of a Flow Perfusion Bioreactor System for Bone Tissue-Engineering Applications. Tissue Eng. 2003; 9:549-54. [PubMed: 12857422] 
48. Amsden B. Solute Diffusion within Hydrogels. Mechanisms and Models. Macromolecules. 1998; 31:8382-95.

49. Knapik DM, Harris JD, Pangrazzi G, Griesser MJ, Siston RA, Agarwal S, et al. The Basic Science of Continuous Passive Motion in Promoting Knee Health: A Systematic Review of Studies in a Rabbit Model. Arthroscopy: The Journal of Arthroscopic \& Related Surgery. 2013; 29:1722-31.

50. Edouard P, Rannou Fo, Coudeyre E. Animal evidence for hyaluronic acid efficacy in knee trauma injuries. Review of animal-model studies. Physical Therapy in Sport. 2013; 14:116-23. [PubMed: 23506791]

51. Huey D, Sanchez-Adams J, Willard V, Athanasiou K. Immunogenicity of bovine and leporine articular chondrocytes and meniscus cells. Tissue Eng Part A. 2012; 18:568-75. [PubMed: 21942992]

52. Holland TA, Bodde EWH, Baggett LS, Tabata Y, Mikos AG, Jansen JA. Osteochondral repair in the rabbit model utilizing bilayered, degradable oligo(poly(ethylene glycol) fumarate) hydrogel scaffolds. Journal of Biomedical Materials Research Part A. 2005; 75A:156-67. [PubMed: 16052490]

53. Mo X-T, Guo S-C, Xie H-Q, Deng L, Zhi W, Xiang Z, et al. Variations in the ratios of co-cultured mesenchymal stem cells and chondrocytes regulate the expression of cartilaginous and osseous phenotype in alginate constructs. Bone. 2009; 45:42-51. [PubMed: 18708174]

54. Grimaud E, Heymann D, Rédini F. Recent advances in TGF- $\beta$ effects on chondrocyte metabolism: Potential therapeutic roles of TGF- $\beta$ in cartilage disorders. Cytokine Growth Factor Rev. 2002; 13:241-57. [PubMed: 12486877]

55. Elder BD, Athanasiou KA. Systematic assessment of growth factor treatment on biochemical and biomechanical properties of engineered articular cartilage constructs. Osteoarthritis and Cartilage. 2009; 17:114. [PubMed: 18571441]

56. Sailor LZ, Hewick RM, Morris EA. Recombinant human bone morphogenetic protein-2 maintains the articular chondrocyte phenotype in long-term culture. J Orthop Res. 1996; 14:937-45. [PubMed: 8982137]

57. Acharya C, Adesida A, Zajac P, Mumme M, Riesle J, Martin I, et al. Enhanced chondrocyte proliferation and mesenchymal stromal cells chondrogenesis in coculture pellets mediate improved cartilage formation. J Cell Physiol. 2011; 227:88-97. [PubMed: 22025108]

58. Liu X, Sun H, Yan D, Zhang L, Lv X, Liu T, et al. In vivo ectopic chondrogenesis of BMSCs directed by mature chondrocytes. Biomaterials. 2010; 31:9406-14. [PubMed: 21056466] 
(a)

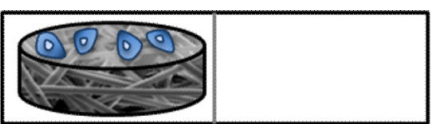

Chondrocytes Alone

AC 35

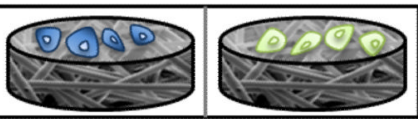

Indirect Co-culture

iAC 35 and iMSC 35

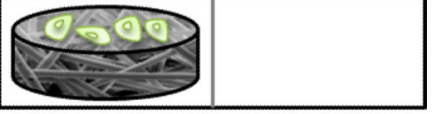

MSCs Alone

MSC 35

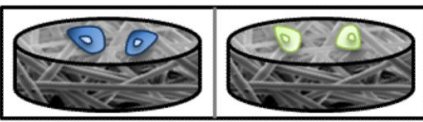

Indirect Co-culture

IAC $\mathbf{1 7 . 5}$ and IMSC $\mathbf{1 7 . 5}$

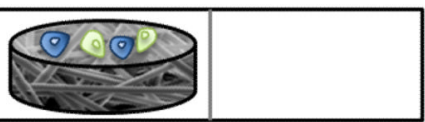

Direct Co-culture

dAC:MSC

(b)

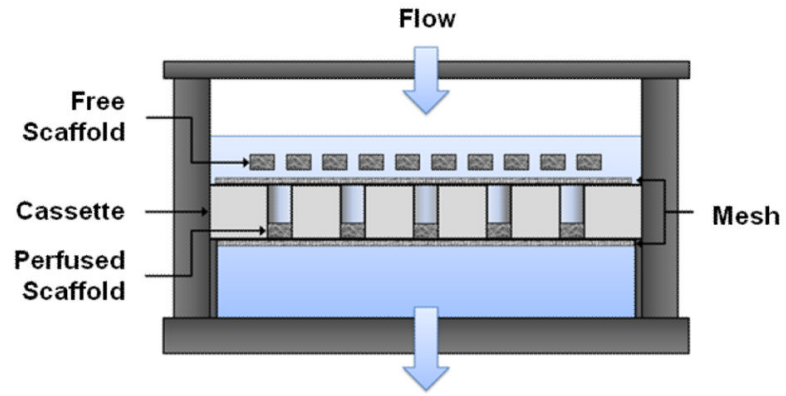

Figure 1.

(a) Schematic indicating seeding and culture arrangement for experimental groups. Divided rectangles are used to represent polypropylene mesh divided wells for static culture. Similar pairings were used in bioreactor culture with scaffolds on the left confined in perfusion cassettes and scaffolds on the right or blank PCL scaffolds included above the cassette as free scaffolds. (b) Bioreactor schematic with free and perfused scaffolds allowing for indirect co-culture under flow conditions. 

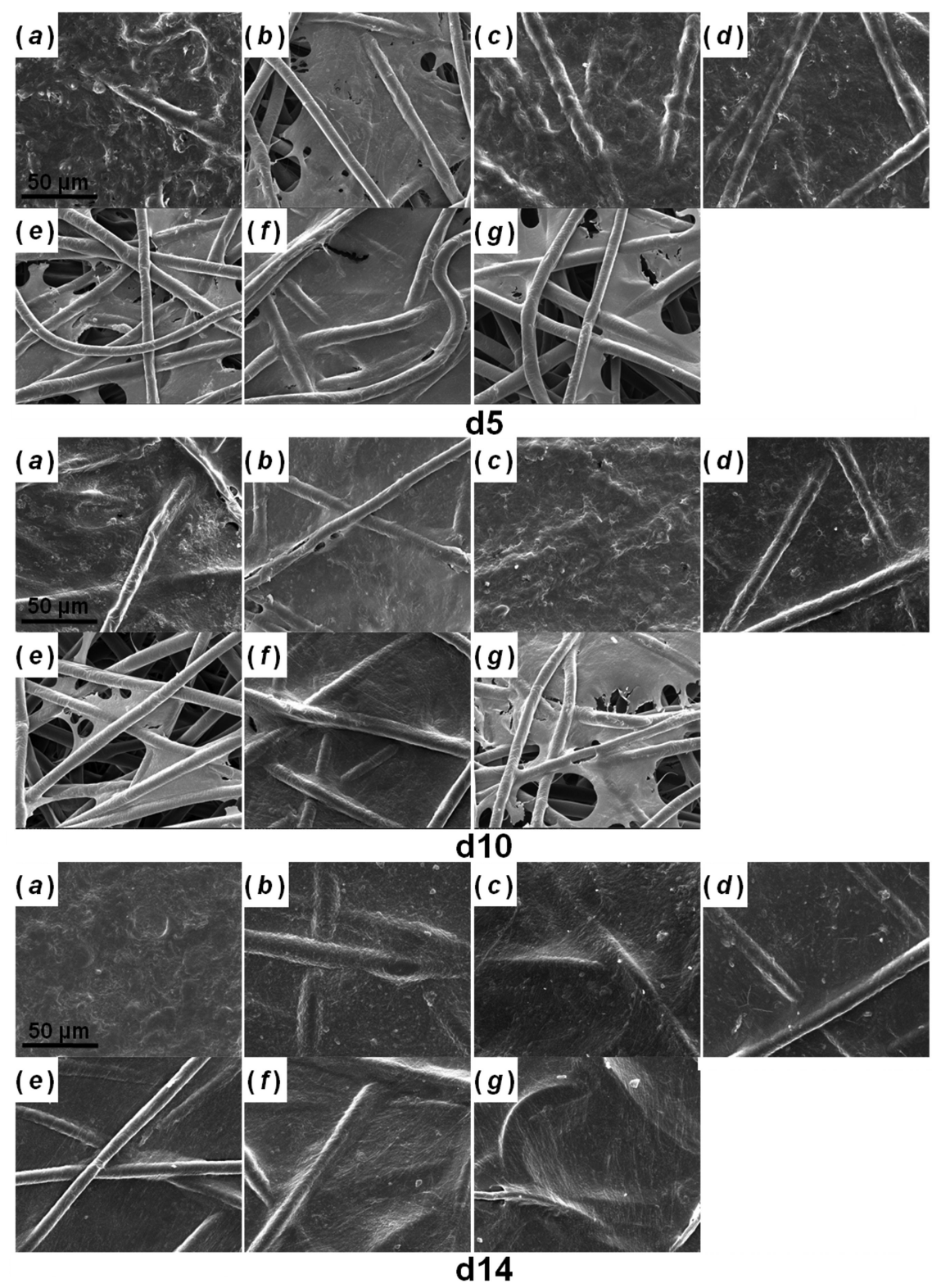

Figure 2.

SEM images depicting the surface morphology of samples cultured in the bioreactor for 5, 10, and 14 days. (a) AC 35, (b) iAC 35, (c) iAC 17.5, (d) dAC:MSC, $(e)$ MSC 35, $(f)$ iMSC 35, $(g)$ iMSC 17.5. Scale bars represent $50 \mu \mathrm{m}$. Magnification 1000x for all images. 

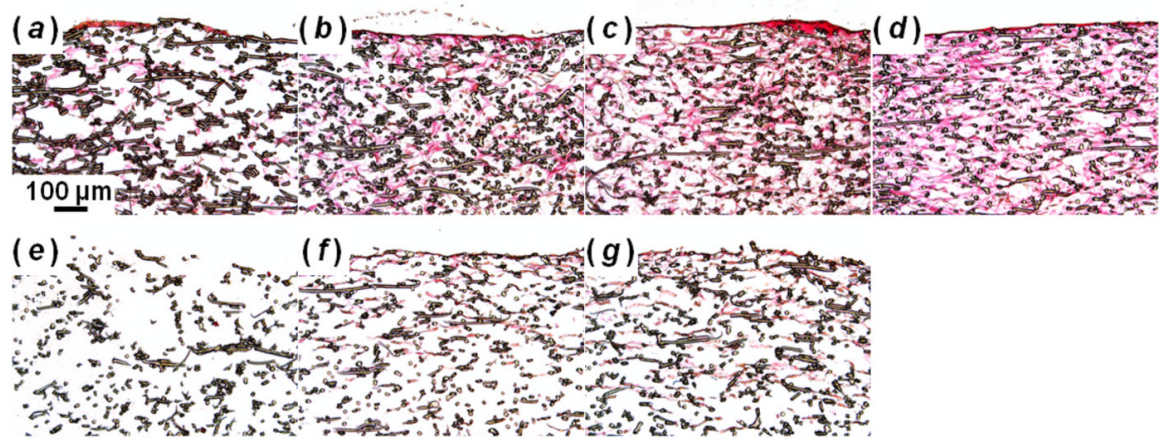

d14
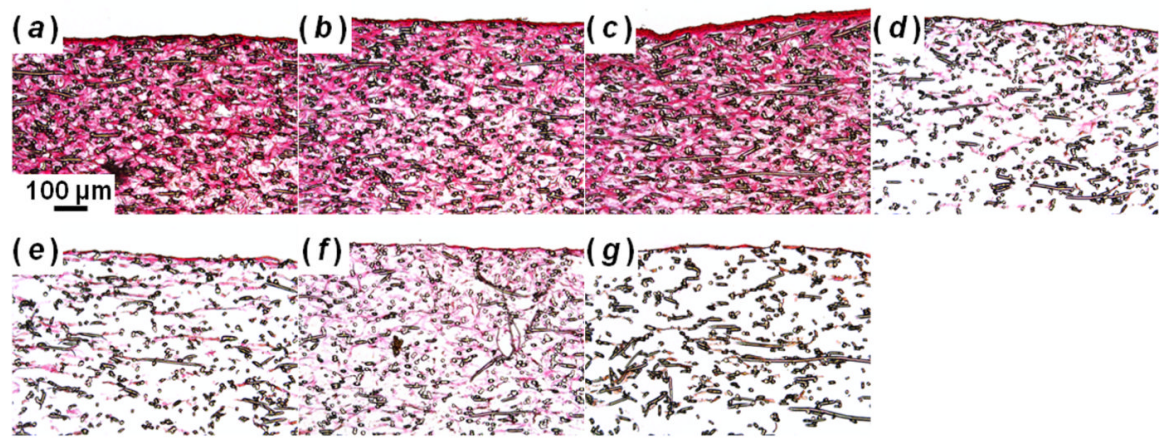

d21

Figure 3.

Histological sections of static samples stained with Picro Sirius Red. Red staining depicts localization of collagen deposits in scaffolds after 14 and 21 days of static culture. (a) AC 35, (b) iAC 35, (c) iAC 17.5, (d) dAC:MSC, (e) MSC 35, (f) iMSC 35, (g) iMSC 17.5. Scale bars represent $100 \mu \mathrm{m}$. Magnification 1000x for all images. 

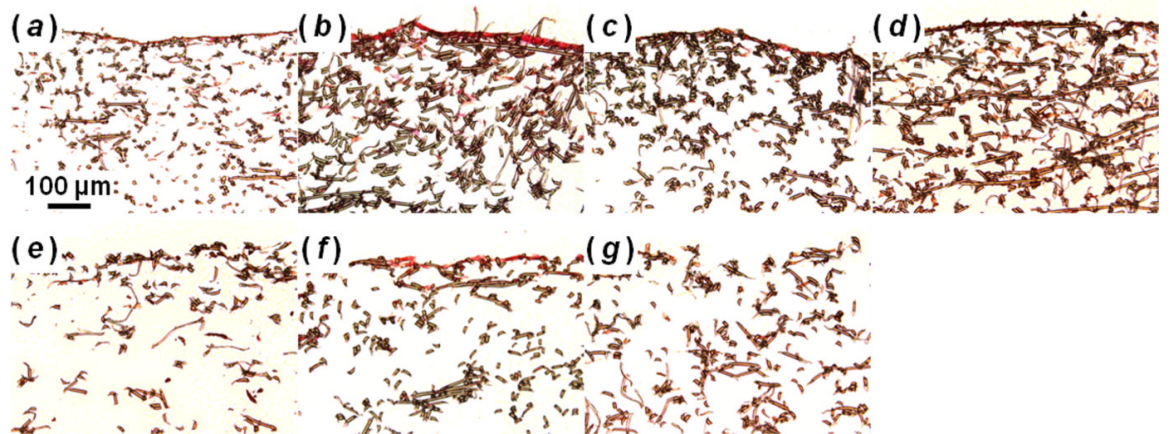

d5
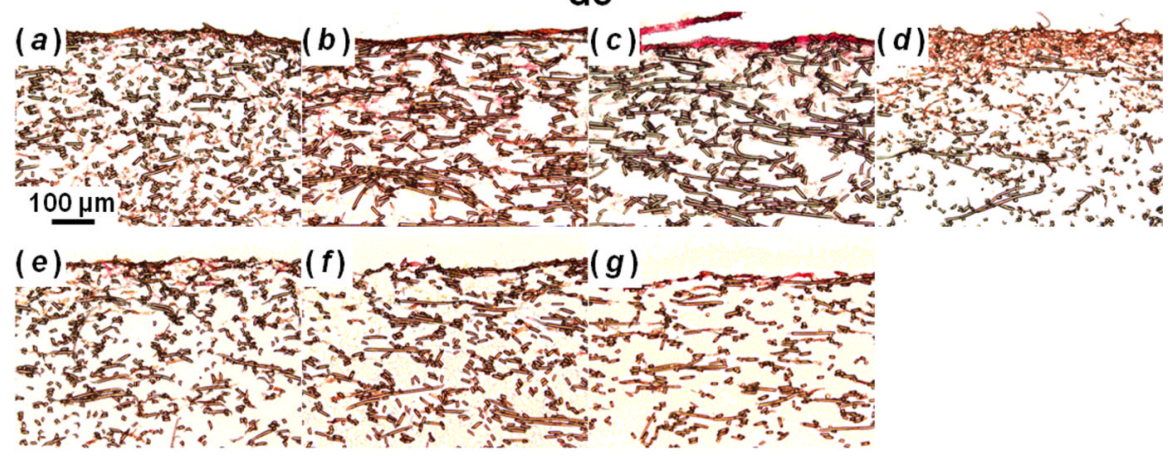

d10
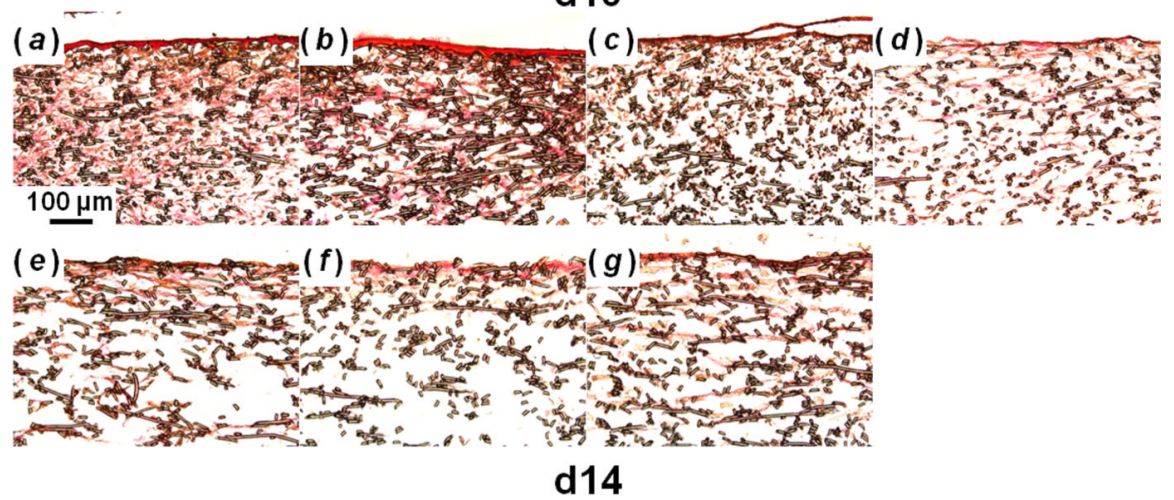

Figure 4.

Histological sections of bioreactor samples stained with Picro Sirius Red. Red staining depicts localization of collagen deposits in scaffolds after 5, 10, and 14 days of bioreactor culture. (a) AC 35, (b) iAC 35, (c) iAC 17.5, (d) dAC:MSC, (e) MSC 35, (f) iMSC 35, (g) iMSC 17.5. Scale bars represent $100 \mu \mathrm{m}$. Magnification 1000x for all images. 

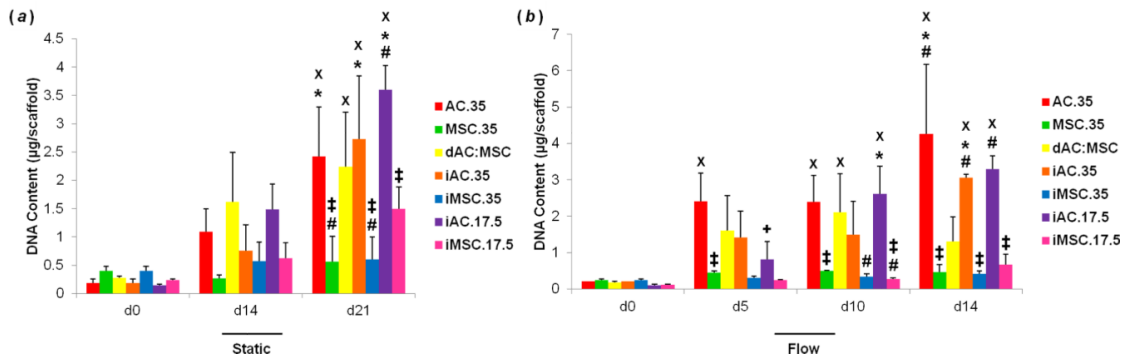

Figure 5.

DNA content for (a) statically and (b) flow perfusion cultured scaffolds at each time point. Symbols indicating statistical significance are defined in Table 2. $p<0.05$. 

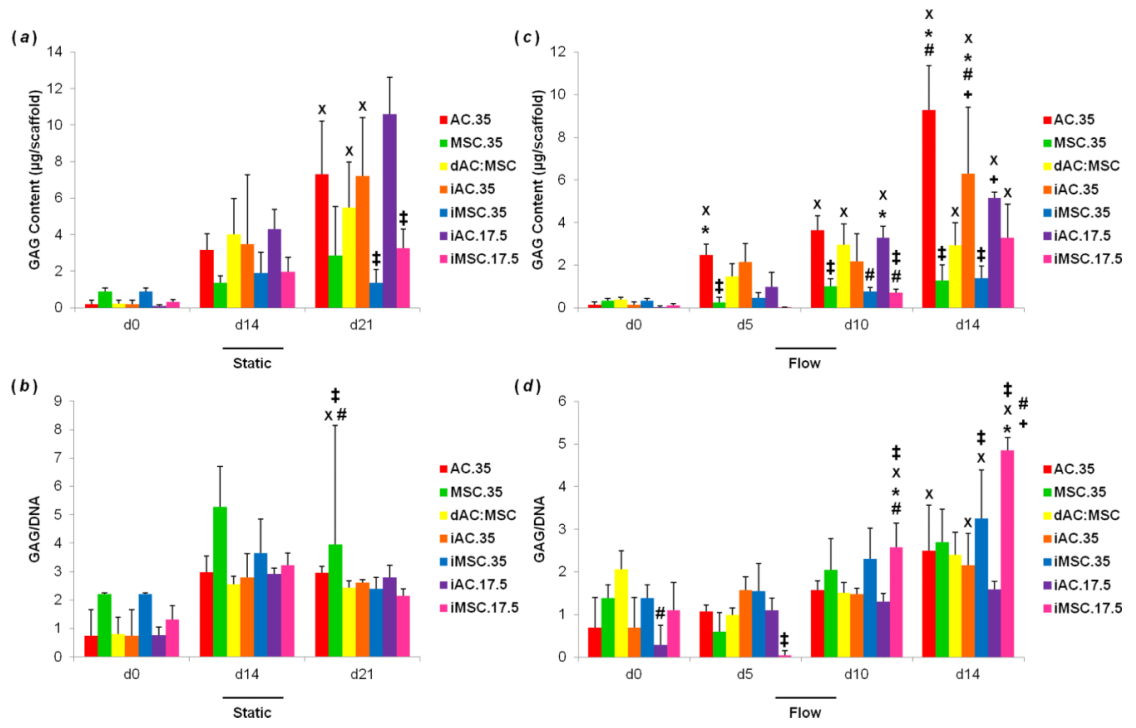

Figure 6.

Characterization of GAG production in static cultures. Total GAG content for (a) statically and (c) flow perfusion cultured scaffolds at each time point. Glycosaminoglycan synthetic activity as normalized to DNA content (b) statically and (d) flow perfusion cultured scaffolds. Symbols indicating statistical significance are defined in Table 2. $p<0.05$. 

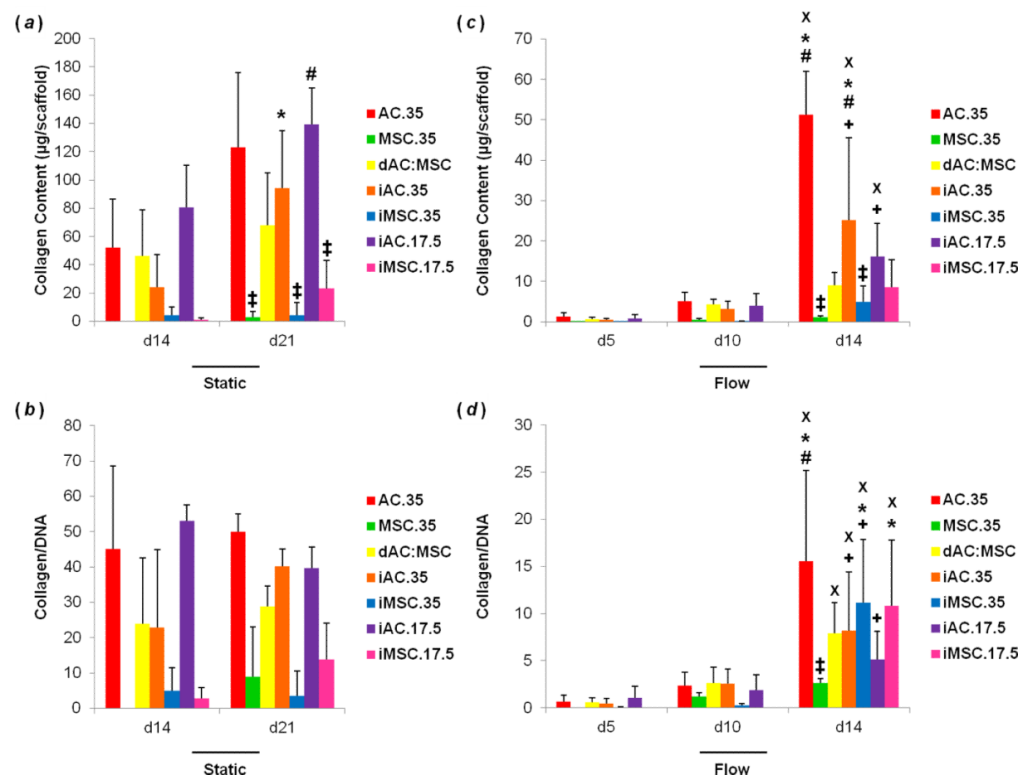

Figure 7.

Characterization of collagen production in static cultures. Total collagen content for (a) statically and (c) flow perfusion cultured scaffolds at each time point. Collagen synthetic activity as normalized to DNA content (b) statically and (d) flow perfusion cultured scaffolds. Symbols indicating statistical significance are defined in Table 2. $p<0.05$. 


\section{Table 1}

Description of experimental groups with cell types and densities seeded.

\begin{tabular}{|ccc|}
\hline Experimental Group & Chondrocytes/scaffold & MSCs/scaffold \\
\hline AC 35 & 35,000 & - \\
\hline MSC 35 & - & 35,000 \\
\hline dAC:MSC & 17,500 & 17,500 \\
\hline iAC 35 & 35,000 & - \\
\hline iMSC 35 & - & 35,000 \\
\hline iAC 17.5 & 17,500 & - \\
\hline iMSC 17.5 & - & 17,500 \\
\hline
\end{tabular}


Table 2

Definition of symbols representing statistical significance. $\mathrm{p}<0.05$.

\begin{tabular}{|ll|}
\hline Symbol & Meaning \\
\hline$\#$ & Statistically significant difference from dAC:MSC group. \\
\hline+ & $\begin{array}{l}\text { Statistically significant difference between an indirectly co-cultured group and chondrocyte-alone (AC 35) or MSC-alone (MSC 35) } \\
\text { group of the same cell type. }\end{array}$ \\
\hline$*$ & Difference between AC and MSC groups cultured similarly. Significance is indicated above the MSC containing counterpart only. \\
\hline$\times$ & Statistically significant difference from the corresponding initial time point. \\
\hline$*$ & Statistically significant difference from the directly preceding time point. \\
\hline
\end{tabular}

